\title{
relazioni
}

\section{SESSIONE 2}

\section{Il Laboratorio di Microbiologia e la gestione delle infezioni in ambito nosocomiale e comunitario}

\author{
Martedì 19 Settembre 2006, ore 14.00 - 18.00, Sala GIALLA
}

\section{S2.1}

\section{RUOLO E RESPONSABILITÀ DEL LABORATORIO DI MICROBIOLOGIA}

\section{Marchiaro $\mathbf{G}$. \\ S.C. Microbiologia, ASO S. Giovanni Battista, Torino}

I profondi cambiamenti in corso nelle organizzazioni sanitarie dei paesi industrializzati, caratterizzati soprattutto dalla deospedalizzazione precoce, dallo sviluppo della Day Surgery e del Day Hospital, dall'incremento delle strutture di lungodegenza e di riabilitazione, estendono i problemi della gestione delle infezioni all'ambiente extraospedaliero e determinano uno stretto legame tra i problemi presenti all'interno e all'esterno dei nosocomi.

A questo si devono aggiungere le emergenze sanitarie a cui ci hanno abituato i mezzi di informazione, la globalizzazione e le esigenze dei cittadini di avere risposte rapide e convincenti.

Alla luce, dunque, di una realtà profondamento mutata il Laboratorio di Microbiologia clinica (LMC) deve affrontare problemi organizzativi nuovi, sviluppare nuove competenze e svolgere un ruolo di riferimento nel Servizio Sanitario.

In particolare in ambito ospedalero, è noto che LMC non si limita al solo compito diagnostico e di indirizzo per la terapia antinfettiva, ma il suo ruolo si articola in attività più complesse quali la sorveglianza e il controllo degli "alert organism", lo studio delle epidemie, la sorveglianza delle antibioticoresistenze. Queste attività non dovrebbero differire sostanzialmente dai nuovi compiti che vengono richiesti anche sul territorio.

La responsabilità di garntire la "qualità del dato microbiologico" impone al LMC, non solo di dotarsi di strumenti tecnologici avanzati, ma di sviluppare strategie di comunicazione che superino i confini dell'Ospedale e giungano negli studi dei Medici di Medicina
Generale e negli ambulatori esterni. Tutta l'esperienza sviluppata in questi decenni per migliorare $\mathrm{i}$ rapporti con i clinici ospedaleri deve essere messa al servizio delle problematiche comunitarie: oltre alle informazioni sui criteri per la scelta delle indagini microbiologiche deve essere adottata anche una reportistica utilizzabile sia per l'approccio diagnostico sia per stimolare azioni di prevenzione e di sorveglianza.

Indispensabili sono gli incontri periodici per definire $\mathrm{i}$ criteri di appropriatezza, incontri che dovrebbero essere contemplati nei programmi di formazione obbligatoria

Nell'ambito della programmazione sanitaria, al fine di migliorare le potenzialità diagnostiche dei laboratori, deve essere previsto un sistema integrato, ospedale/territorio, in cui siano riconosciuti livelli diversi di prestazioni: impegno economico ed elevata tecnologia devono convogliare in Centri di riferimento, collegati via rete con le strutture e i servizi istituzionali.

Pertanto la riorganizzazione dei LMC deve comprendere i seguenti provvedimenti:

- sviluppo dei sistemi informativi;

- individuazione dei Laboratori di riferimento;

- standardizzazione delle procedure e definizione dei livelli di attività;

- istituzione di Gruppi di lavoro con specialisti ospedalieri, medici del territorio, epidemiologi per valutare i problemi che emergono di volta in volta e programmare gli interventi;

- sviluppo di piani di formazione ;

Il ruolo e la responsabilità dei LMC nella gestione delle infezioni nosocomiali e comunitarie rappresenta anche e soprattutto una nuova opportunità per accrescere le conoscenze: l'attività diagnostica tradizionale non è più sufficiente a supportare $\mathrm{i}$ compiti che ci vengono richiesti: è necessario sviluppare processi organizzativi rivolti anche alla sorveglianza e prevenzione sia in ambito ospedaliero che comunitario. 